\title{
Two-Photon Imaging of Microglia in the Mouse Cortex In Vivo
}

\author{
Axel Nimmerjahn
}

Microglia are the primary immune effector cells of the brain parenchyma. They are distributed throughout the brain at various densities. Two-photon fluorescence microscopy, together with expression of fluorescent proteins in microglia, has enabled study of these fascinating cells in vivo. Imaging studies have shown, for example, that microglia continually survey their cellular environment and immediately respond to injury. However, we still know very little about their roles in various parts of the developing and adult brain or their diverse effector functions in aging and different disease states. Experimental procedures have been developed for minimally invasive short- and long-term twophoton imaging of microglial cells in cortical regions of the intact mouse brain. This protocol describes two-photon imaging of microglia in the mouse cortex in vivo, using mice which have had a head plate implanted and have been prepared with either a thinned skull or optical window. Technical pitfalls, limitations, and alternative approaches are also discussed.

MATERIALS

It is essential that you consult the appropriate Material Safety Data Sheets and your institution's Environmental Health and Safety Office for proper handling of equipment and hazardous materials used in this protocol.

RECIPE: Please see the end of this article for recipes indicated by $<R>$. Additional recipes can be found online at http://cshprotocols.cshlp.org/site/recipes.

Reagents

ACSF for two-photon imaging $<\mathrm{R}>$

Alternatively, ACSF can be purchased (e.g., from Harvard Apparatus).

Cx3cr1-eGFP (enhanced green fluorescent protein) knockin mice or Iba1-eGFP transgenic mice The mice should have a head plate implanted as described in Surgical Implantation of a Head Plate in Mice in Preparation for In Vivo Two-Photon Imaging of Microglia (Nimmerjahn 2012a). This protocol also gives details on anesthesia and maintaining the animal during procedures. The mice should also be prepared with either a thinned skull or sealed craniotomy as described in Optical Window Preparation for Two-Photon Imaging of Microglia in Mice (Nimmerjahn 2012b).

Ophthalmic ointment (e.g., Puralube Vet Ointment, PharmaDerm)

Skin disinfectant (e.g., Techni-Care, Care-Tech Laboratories, Inc.)

Surface disinfectant (e.g., Virkon S, DuPont)

Equipment

Dressing, transparent (e.g., Bioclusive, Johnson \& Johnson)

Flexible-arm magnetic-base holder (McMaster-Carr)

Adapted from Imaging in Neuroscience (ed. Helmchen and Konnerth). CSHL Press, Cold Spring Harbor, NY, USA, 2011.

(C) 2012 Cold Spring Harbor Laboratory Press

Cite this article as Cold Spring Harb Protoc; 2012; doi:10.1101/pdb.prot069294 
Glass bead sterilizer (Dent-EQ model BS-500)

Head holder, custom-made, $6.4 \times 76 \times 6.4-\mathrm{mm}(\mathrm{W} \times \mathrm{L} \times \mathrm{H})$, stainless steel (Fig. 1)

Image-processing resources:

- ImageJ (e.g., for lateral image alignment, projection images)

- Custom-written software (e.g., using IGOR Pro from WaveMetrics, Inc.)

- Commercial software packages (e.g., Amira from Visage Imaging or Imaris from Bitplane)

Imaging setup:

- Two-photon microscope (custom-made) equipped with an ultrafast Ti-sapphire laser, a 10-W all-solid-state pump laser (Spectra-Physics), a motorized micromanipulator-controlled (Sutter Instruments) $\mathrm{x}-\mathrm{y}$ table (New England Affiliated Technologies, Inc.), a motorized objective focusing nose piece (e.g., using a Vexta stepping motor), and one or more fluorescence detection channels (filter sets depend on application; for excitation/ emission spectra of common fluorescent proteins, see Spiess et al. 2005)

- Electro-optic laser power controller (e.g., model 302RM amplifier and 350-80-LA modulator, Conoptics)

- Beam shutter (e.g., Thorlabs SH05)

- Galvanometer-based optical scanner (e.g., Cambridge Technology 6215H)

- High-numerical-aperture (NA) water-immersion objective (e.g., Zeiss Achroplan 40x $0.80 \mathrm{NA}$ )

- Variable-gain, low-noise current amplifiers (e.g., Femto DLPCA-200)

- High-speed digital signal integrator, custom-made (per Nguyen et al. 2006)

A
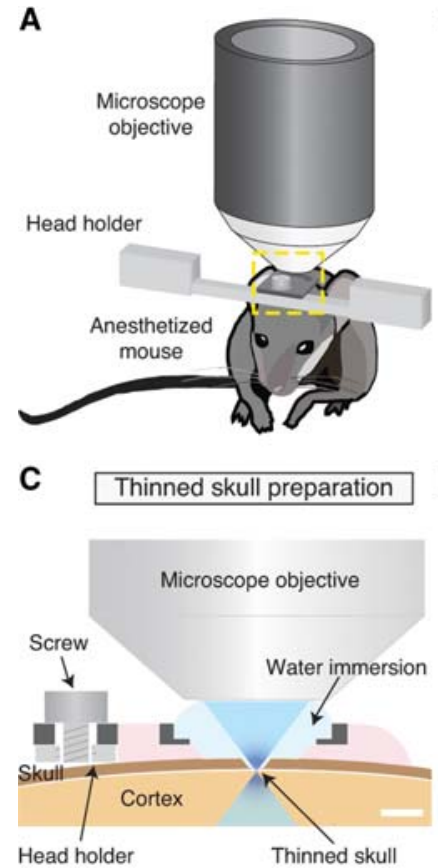
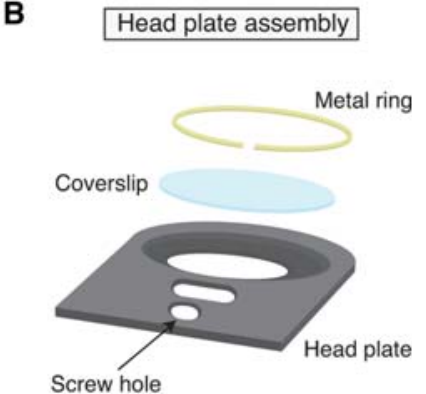

Open skull preparation

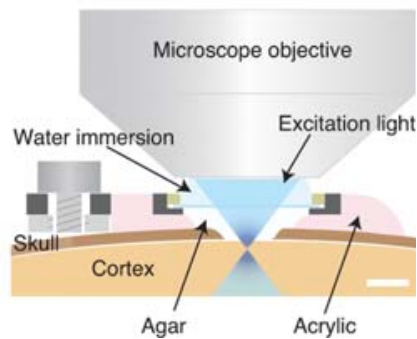

FIGURE 1. Experimental procedures for two-photon fluorescence imaging in the cortex of anesthetized mice. $(A)$ Schematic view of experimental setup. Stable fluorescence imaging is enabled by restraining the animal's head via a head plate and head holder. A blow-up of the head plate (yellow dashed box) is shown in $(B)$. (B) Firm and repeatable head restraint is facilitated by implanting a lightweight head plate. Coverslip and metal ring are used to stabilize and seal open skull preparations. $(C, D)$ Lateral view showing how skull, head plate, and head holder interface with each other. Scale bar, $2 \mathrm{~mm}$. (C) Schematic view of a thinned skull preparation. A restricted area of the skull (typically $<350$ $\mu \mathrm{m}$ in diameter) is reduced to an $\sim 25$ - to 35- $\mu \mathrm{m}$-thin layer. Cells and their processes can be visualized over short and long timescales up to a depth of $\sim 250 \mu \mathrm{m}$. (D) Open skull preparation. To reduce heartbeat- and breathing-induced motion artifacts as well as optical aberrations, the exposed brain is covered with agarose gel and a coverslip. This preparation is particularly useful for short-term investigation of deep cortical regions and large contiguous brain areas. 
A. Nimmerjahn

- Data acquisition hardware (e.g., items NI PCI-6110, NI BNC-2110, NI SHC68-68-EP, National Instruments)

- Data acquisition software (e.g., MPScope; see Nguyen et al. 2006)

- Optical power meter (e.g., Thorlabs FM203)

- Cold light source (e.g., Schott KL 1500)

- Digital or charge-coupled device (CCD) camera (e.g., from Andor Technology)

- Infrared viewer (e.g., FIND-R-SCOPE, FJW Optical Systems, Inc.) or viewing card (Thorlabs, Inc.)

Laboratory animal anesthesia system (VetEquip Inc. 901806), anesthetic gas (isoflurane from

Southmedic, Inc.), carrier gas tank (e.g., medigrade oxygen from Praxair)

A waste anesthetic gas system (VetEquip Inc. 933101) is also recommended.

Optical power meter (Thorlabs FM203)

Protective eyewear (to prevent laser damage)

Stereomicroscope (Leica MZ12.5)

Stereotaxic apparatus/dissecting setup (custom-made or from David Kopf Instruments)

Surgical eye spears (e.g., Henry Schein Medical 1556455)

Temperature regulation system with heating pad and rectal probe (FHC Inc. 40-90-8, 40-90-5, 40-902-07)

\section{METHOD}

All procedures described here were approved by the Max Planck Society's and Stanford's Institutional Animal Care and Use Committee (IACUC). The initial imaging session typically takes $\sim 1.5-2 \mathrm{~h}$ but up to $5 \mathrm{~h}$ depending on the number of recording sites and biological processes investigated. Subsequent imaging sessions last $\sim 90$ min or less.

\section{Two-Photon Imaging}

1. Tune laser to desired wavelength. The following imaging parameters are useful guidelines:

- Excitation wavelength: $\sim 950 \mathrm{~nm}$ (this wavelength is optimal for eGFP excitation; see Spiess et al. 2005)

- Excitation intensity at sample surface: typically, $<15 \mathrm{~mW}$ (depending on details of the optical setup, animal age, surgical preparation, imaging depth, etc.)

- Images/frame rate: $512 \times 512$ pixels $(4.07 \mathrm{~Hz}), 256 \times 256$ pixels $(10.17 \mathrm{~Hz})$

- Average: typically one to two frames

- Recording duration: minutes to months (depending on biological process investigated)

- Sampling interval: seconds to days (depending on biological process investigated and volume sampled)

2. Make sure the back aperture of the objective is as evenly illuminated as possible. Use an infrared viewer or infrared viewing card to visualize the laser beam. Adjust microscope mirrors as necessary. Wear appropriate protective eyewear and use minimal laser power during this process.

3. Using an optical power meter, measure the average laser power exiting the microscope objective for different beam deflector settings. Excessive tissue illumination can cause microglia activation. During imaging keep average laser power levels well below the previously determined photodamage threshold (see Discussion).

4. Transfer animal to microscope. Using adjustments on the stereotaxic apparatus, tilt the animal's head such that the head plate's upper surface is perpendicular to the optical axis. If imaging at later time points is required, mark stereotaxic position and settings. Alternatively, position a laser pointer inside the imaging setup such that its beam reflects off a reflective particle/area on 
Downloaded from http://cshprotocols.cshlp.org/ on April 26, 2023 - Published by

the head plate into a target area. Keep laser pointer and target area fixed for precise head alignment during subsequent imaging sessions.

5. Using the microscope's ocular (or camera) and cold light illumination, locate the brain surface by translating the microscope stage or objective. Switch to fluorescence mode.

6. Start imaging using minimal excitation intensity. If transcranial imaging is performed, assess skull thickness by measuring the distance at which autofluorescence of the skull is observed. For a skull thickness of $\sim 25-35 \mu \mathrm{m}$, fluorescently labeled cells and their fine processes should be resolved up to a depth of $\sim 200 \mu \mathrm{m}$ below the pia (Fig. 2).

7. Identify an area of interest. Take a low-magnification but high-resolution fluorescence image stack for later reference.

8. Switch back to cold light illumination. Inspect corresponding surface blood vessel patterns. Mark the recording site location on the high-resolution surface blood vessel map recorded earlier (see Method B, Step 7 of Optical Window Preparation for Two-Photon Imaging of Microglia in Mice [Nimmerjahn 2012b]). Set micromanipulator coordinates to 0.

9. Switch back to fluorescence mode. Acquire imaging data as necessary. Keep the following points in mind:

i. Record micromanipulator coordinates relative to the brain surface and other microscope settings.

ii. For additional recording sites, repeat Steps 7-9.

iii. During prolonged imaging sessions, monitor animal periodically and treat as necessary (e.g., inject warm fluids or adjust anesthetic level). See Troubleshooting.
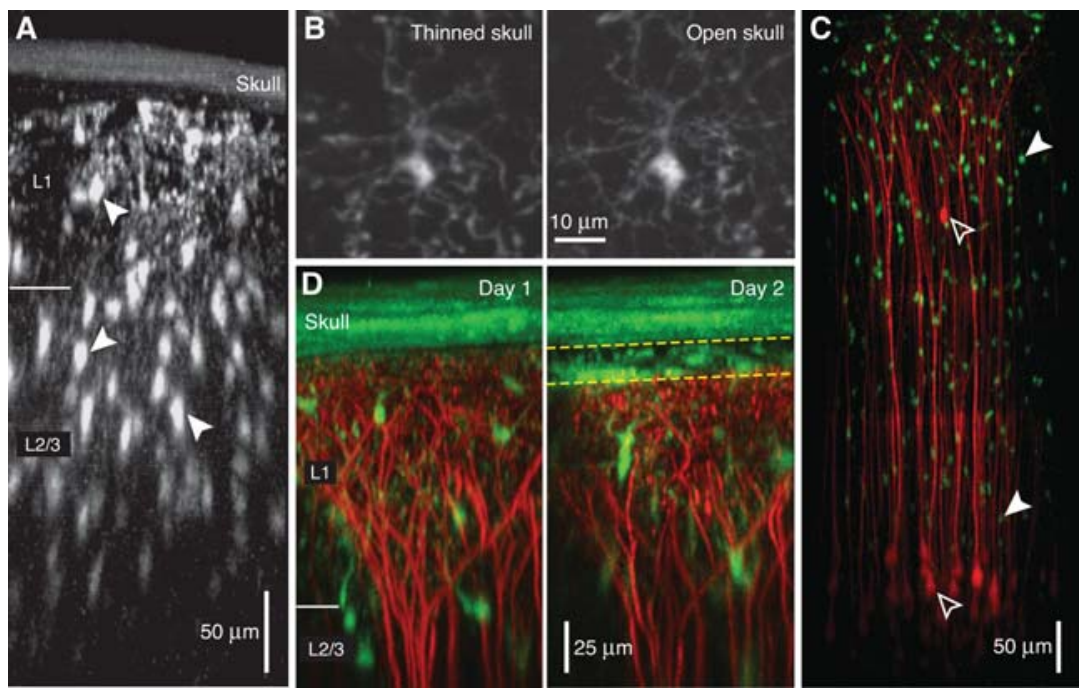

FIGURE 2. Selected aspects of thinned and open skull preparations. (A) Example image showing a maximum-intensity side projection of eGFP-positive cells in the neocortex of a heterozygous Cx3cr1-eGFP mouse. Images were acquired through the thinned, autofluorescent skull (visible on top). Note that microglia somata (closed arrowheads) and their fine processes can be resolved clearly in layer 1 (L1) and parts of layer 2/3 (L2/3). Image quality degrades rapidly with depth. (B) (Left) Fluorescence images showing a transcranially imaged microglial cell in L1. (Right) The same microglial cell after skull removal. Fine processes can be resolved in both cases. (C) Example image showing a maximumintensity side projection of enhanced yellow fluorescent protein (eYFP)-expressing neurons (red, open arrowheads) and eGFP-positive microglia (green, closed arrowheads) in the neocortex of a Thy1-eYFP $\times$ Cx3cr1-eGFP mouse implanted with an open skull window. Note increased depth penetration and spatial resolution compared to $(A)$. (D) (Left) Side projection showing neuronal dendrites (red) and microglial cells (green) in the neocortex of a Thy 1eYFP $\times$ Cx3cr1-eGFP mouse immediately after thinned skull preparation. (Right) The same tissue volume imaged $1 \mathrm{~d}$ later without skull rethinning. Improper skull thinning can lead to inflammation in regions near the skull surface (yellow dashed lines). (A, B, Modified from supplementary online material from Nimmerjahn et al. 2005.) 
A. Nimmerjahn

10. At the end of a thinned skull imaging session, treat the animal as follows:
i. Dry and disinfect the skull surface (e.g., using surgical eye spears and Techni-Care, respectively).
ii. Cover the head plate surface (e.g., using a transparent dressing).
iii. Detach head plate from head holder.
iv. Return the animal to its cage. Keep it on a heating pad and monitor until it is awakening.

11. Clean the microscope's objective and record transmitted average laser power for all beam deflector settings used during the experiment.

12. Thoroughly disinfect all areas and instruments (e.g., with a glass bead sterilizer to autoclave tips of surgical instruments).

Two-Photon Imaging at Later Time Points

13. Transfer the animal to an anesthesia induction chamber. Anesthetize the animal with gaseous isoflurane $\left(3 \%\right.$ in $\left.1 \mathrm{~L} / \mathrm{min}_{2}\right)$.

Gaseous isoflurane is particularly useful for juvenile mice and long-term investigations because it allows control of anesthesia level and duration without the need for repeated anesthetic injections.

14. Place the animal in the stereotaxic apparatus used previously. Fix head plate to head holder. Maintain animal's body temperature using a rectal probe and heating pad. Assess animal condition periodically and treat as necessary.

See Troubleshooting.

15. Protect the animal's eyes with ophthalmic ointment.

16. Remove head plate cover (Step 10). Inspect optical window condition under a stereomicroscope. Clean/rinse skull and optical window surface.

17. Transfer animal to microscope. Adjust head position as necessary (see Step 4).

18. Locate previous recording sites using the reference image(s)/stack(s)/coordinates recorded during the first imaging session (see Method B, Step 7 of Optical Window Preparation for Two-Photon Imaging of Microglia in Mice [Nimmerjahn 2012b], and Steps 7 and 9 above). If transcranial imaging is performed, skull rethinning may be necessary (see Discussion).

See Troubleshooting.

19. Acquire imaging data as necessary (Fig. 3; see Movies 1-3 online at http://cshprotocols.cshlp. org). If possible, use the same microscope settings as in previous session(s).

See Troubleshooting.

20. At the end of the imaging session, dry and disinfect skull/optical window surface and cover head plate surface as in Step 10.

21. Detach head plate from head holder and return animal to its cage.

22. Clean the microscope's objective and record transmitted average laser power for all beam deflector settings used during the experiment.

23. Thoroughly disinfect all areas and instruments.

Problem (Step 9): Motion artifacts occur during imaging, particularly at high magnification.

Solution: In anesthetized animals, motion artifacts during imaging can arise from heartbeat or breathing, leading to micrometer-scale displacements of the brain relative to the anchored skull. Consider the following: 

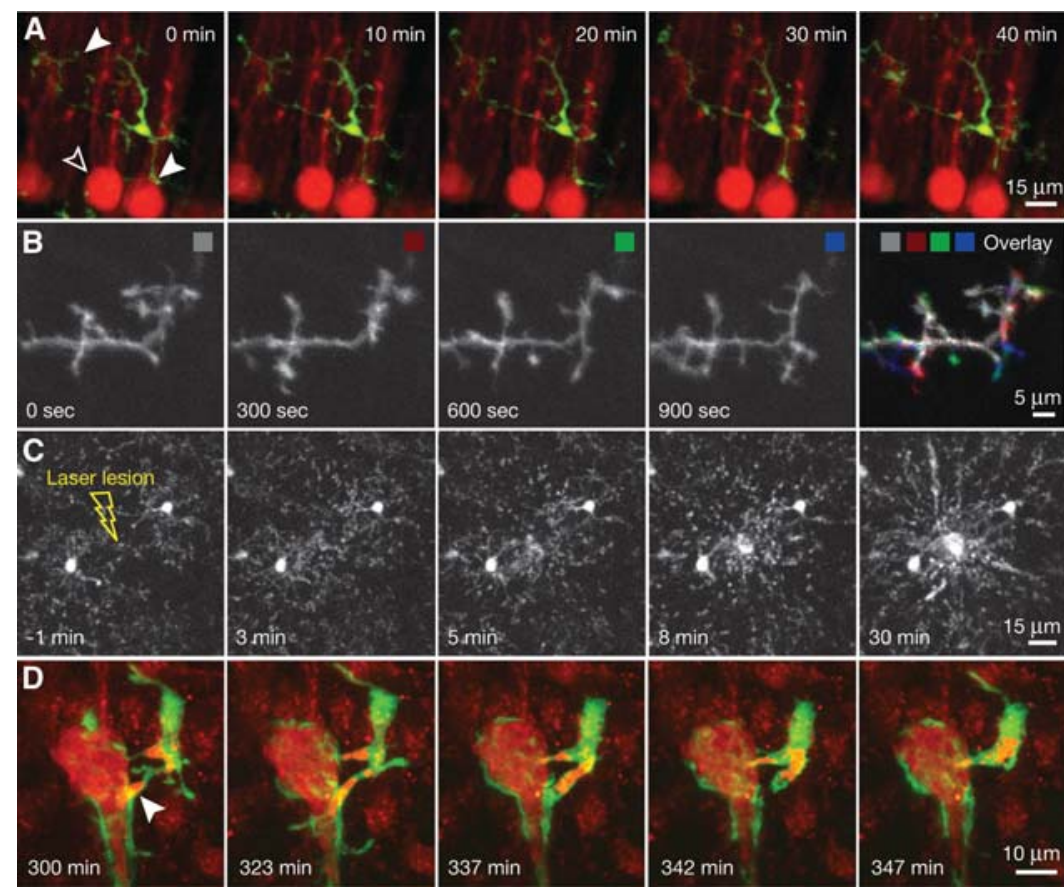

FIGURE 3. Microglia dynamics in the healthy and injured adult brain. (A) Overlay images from a time-lapse recording showing eGFP-expressing microglia (green) and eYFP-expressing Purkinje cells (red, open arrowhead) in the cerebellar cortex of an anesthetized Thy 1 -eYFP $\times$ Cx3cr1-eGFP mouse. Images were recorded near a bend in the cerebellar folium, which allowed the molecular and Purkinje cell layers to be visualized within a single image. Note that microglial processes (closed arrowheads) are highly motile. Neurons appear structurally stable during the same time interval. $(B)$ Image frames showing a microglial process at high magnification. Fine protrusions continually palpate the cellular environment. The image on the far right is an overlay of the four images on the left pseudocolored in gray, red, green, and blue. (C) Microglia response to laser lesion. Within minutes, microglia extend their processes toward the lesion site where they shield injured tissue. Time before and after laser lesion is indicated. $(D)$ Phagocytosis of cellular debris by microglia. Overlay images showing a mechanically injured Purkinje cell (red) braided by microglia processes. Time after mechanical injury is indicated. Sulforhodamine 101 was used to stain Bergmann glia and injured cell bodies. $(C$, Images are based on data from supplementary online material from Nimmerjahn et al. 2005.)

1. To minimize motion artifacts in an open skull preparation, craniotomy size should be kept small $(<1.5 \mathrm{~mm})$.

2. To further dampen brain motion, increase agar viscosity.

3. Exerting increased pressure onto the brain via the coverslip is not recommended because of the risk of causing tissue damage.

4. In time-lapse recordings, image stability may be improved by triggering image acquisition from the animal's heartbeat or breath.

5. Changing the animal's body position or anesthetic level may help to diminish breathing-induced motion artifacts.

6. Higher scan rates (at lower resolution) may help to reduce within-frame image distortions.

7. Lateral motion artifacts remaining in and between recorded images can be largely corrected offline by image registration algorithms. However, correction of axial motion artifacts remains challenging. See Dombeck et al. (2007), Greenberg and Kerr (2009), and Nimmerjahn et al. (2009) for example algorithms.

Problem (Step 9): Microglia become activated during two-photon imaging.

Solution: Consider the following:

1. Use as little laser light exposure as possible. 
2. If standard microscope settings are insufficient to achieve adequate signal-to-noise ratio, fluorescence excitation or detection efficiency can be boosted using, for example, high-NA objectives in combination with large-aperture detection optics (Oheim et al. 2001), dispersion precompensation (Tang et al. 2006), supplementary fiber-optic light collection (Engelbrecht et al. 2009), passive pulse splitting (Ji et al. 2008), bunched pulse excitation (Donnert et al. 2009), or adaptive wave-front correction (Rueckel et al. 2006).

3. Some improvement in signal-to-noise ratio may be achieved offline using computational methods (e.g., blind image deconvolution).

Problem (Step 14): The head plate detaches from the skull during head fixation.

Solution: This is a highly unlikely scenario that may be caused by improper head plate implantation or mouse handling during head fixation. To improve the adhesion of dental cement to the skull in Step 15 of Surgical Implantation of a Head Plate in Mice in Preparation for In Vivo Two-Photon Imaging of Microglia [Nimmerjahn 2012a], apply a thin layer of cyanoacrylate adhesive (e.g., Vetbond) to the skull surface in Step 13 of that protocol, keeping the area over which the optical window will be performed uncovered. Use great care when restraining the animal's head. Avoid shear stress.

Problem (Step 18): Compared to previous imaging sessions, microglia underneath the thinned skull optical window appear activated.

Solution: Microglia activation may be caused by improper surgical preparation (Fig. 2D). Using postmortem histology, assess the extent of glial activation in relation to optical window position. In future preparations, thin less, avoid pushing the skull toward the cortical surface, or allow sufficient heat dissipation.

Problem (Step 18): Using the same settings as in previous imaging sessions, transcranially acquired fluorescence images appear dimmer or more distorted.

Solution: Assuming this is not caused by light collection problems, for example, from low water immersion or bubbles in water immersion, reduced fluorescence levels/image distortions may be caused by skull regrowth. Consider the following:

1. Rethinning may improve optical conditions (see Discussion).

2. Low fluorescence levels may be compensated by increasing excitation intensity, although at the increased risk of causing photodamage.

Problem (Step 19): The imaged volume appears rotated compared to previous imaging sessions (e.g., because of imprecise head alignment).

Solution: Compensate image rotation online as much as possible (see Step 5). Remaining image rotations can be largely corrected offline (using ImageJ or commercial software packages such as Amira).

Microglia form the brain's first line of defense against injury and infection. They are highly sensitive to even slight changes in their cellular environment. Disturbances of central nervous system homeostasis lead to rapid changes in microglia phenotype (Raivich et al. 1999; Ransohoff and Perry 2009). Studying normal microglia physiology in vitro has therefore been challenging, given the disturbances that cell isolation and tissue slicing introduce.

Various techniques, including bioluminescence imaging, magnetic resonance imaging, positron emission tomography, and two-photon microscopy, now enable minimally invasive study of microglia in the intact brain (Wiart et al. 2006; Lalancette-Hebert et al. 2009; Ransohoff and Perry 2009). Two-photon microscopy is presently the only technique that provides submicrometer spatial and 
subsecond temporal resolution for the cellular-level study of microglia physiology in vivo (GarciaAlloza and Bacskai 2004). Owing to its superior resolution, pathological changes can be detected and followed at much earlier stages than with other minimally invasive techniques (Garcia-Alloza and Bacskai 2004). However, given its restricted depth penetration, two-photon imaging is limited to several hundred micrometers below the cortical surface (Helmchen and Denk 2005). Nevertheless, the ability to image with single-cell resolution in the intact brain is a powerful tool for studying the role of microglia in health and disease.

\section{Choosing an Optical Window Type}

Two-photon imaging of cellular processes in the brain of live mice involves creation of an optical window. This window can either be a thinned skull region or a sealed craniotomy, as described in Optical Window Preparation for Two-Photon Imaging of Microglia in Mice (Nimmerjahn 2012b). Both thinned skull (Davalos et al. 2005; Nimmerjahn et al. 2005; Kim and Dustin 2006; Wake et al. 2009) and open skull preparations (Davalos et al. 2005; Nimmerjahn et al. 2005; Haynes et al. 2006; Kim and Dustin 2006; Bolmont et al. 2008; Koenigsknecht-Talboo et al. 2008; Mandrekar et al. 2009; Wake et al. 2009) have been used in the study of microglia physiology. Microglia in various parts of the mouse neocortex have been studied, but most of these studies have been restricted to periods of a few hours. Few studies have tracked microglial cells for extended periods (i.e., $>24 \mathrm{~h}$ ) (Kim and Dustin 2006; Bolmont et al. 2008). All studies so far have been conducted under various types of anesthesia.

The choice of optical window type depends on experimental requirements. The preferred method for short- and long-term imaging experiments is the thinned skull preparation because, when performed correctly, it is the least invasive to parenchymal tissue. However, some applications may require the use of an open skull window. Skull removal provides direct access to the brain parenchyma, allowing investigation of molecular mechanisms underlying microglia function by means of pharmacology (Davalos et al. 2005; Nimmerjahn et al. 2005; Haynes et al. 2006). Other considerations in choosing optical window type are desired imaging depth, spatial resolution, and cortical area to be monitored. Compared to an open skull preparation, the thinned skull window provides somewhat lower spatial resolution and depth penetration into tissue (Helm et al. 2009). The extent to which these parameters are affected depends on the thickness and texture of the bone remaining after the thinning procedure. Most texture and thickness irregularities can be eliminated by careful scraping with dedicated surgical blades. Remaining irregularities in skull thickness can lead to image distortions (Helm et al. 2009), some of which may be diminished, for example, using adaptive wave-front correction (Rueckel et al. 2006). However, because the risk of causing bone cracks increases with skull area to be thinned, imaging of large contiguous cortical areas (several millimeters in diameter) has been challenging. High-resolution two-photon imaging of extensive cortical areas or micrometerscale structures deep inside the cortex $(>250 \mu \mathrm{m})$ may therefore require the use of an open skull preparation (Holtmaat et al. 2009).

Experimenters using an open skull preparation should bear in mind that skull removal may lead to mechanical injuries to the cortical surface or immediate disturbances in local blood perfusion, bloodbrain barrier permeability, and brain homeostasis (Navari et al. 1978; Kawamura et al. 1990). Hence, imaging of microglial cells close to the cortical surface should be avoided. External influences such as temperature gradients between cortical tissue and the water-immersion objective should be minimized by using, for example, an objective heater system. Long-term studies should take into account that opening the cranial cavity can lead to tissue inflammation involving glial activation that may persist for up to several weeks (Bacskai et al. 2001; Xu et al. 2007). Treatment with anti-inflammatory drugs (dexamethasone, carprofen) may reduce tissue reactions (Holtmaat et al. 2009) but may not prevent the transient increase in optical window opacity often seen within the first 1-2 weeks after craniotomy (Xu et al. 2007). Hence, if the use of an open skull preparation is inevitable, control experiments should be performed to ensure that observed cellular processes are nonpathological.

Similar considerations apply to thinned skull preparations. Although parenchymal cells have been imaged successfully over days without apparent signs of inflammation, cellular structures in the 
A. Nimmerjahn

meninges (which lie outside the blood-brain barrier) can be affected by the manual thinning procedure (Kim and Dustin 2006). Thinning approaches that exert minimal pressure onto the skull are desirable, particularly for studies in juvenile mice (postnatal day 12 or younger) whose skull is soft and flexible, and for long-term imaging experiments in which efficient skull rethinning is difficult and typically limited to a few repeats (Holtmaat et al. 2009). Alternative approaches to manual thinning may involve laser ablation (Walter et al. 1999). No matter which thinning approach is used, reducing the bone to a thin layer eliminates bone blood vessels and marrow. This can lead to local bone inflammation or degeneration. Repeated head plate detachment and suturing of the animal's skin during long-term experiments (Christie et al. 2001; Grutzendler et al. 2002) may compromise tissue over time. Chronic implantation of a lightweight head plate (Fig. 1) provides an alternative solution. Head plate implantation allows easy and reproducible head fixation in a manner less stressful to the animal. However, as the skin above the optical window is removed, which normally provides a protective barrier, great care must be taken to avoid infection of or mechanical damage to the optical window.

\section{Potential Effect of Two-Photon Imaging}

Another critical aspect in microglia imaging is the potential effect of two-photon imaging on tissue physiology itself. Excessive illumination can cause pathological tissue changes. Photodamage threshold depends on various parameters, including pulse duration, exposure time, and excitation intensity (Koester et al. 1999; Hopt and Neher 2001) and therefore needs to be determined experimentally. This can be achieved, for example, by exposing stained cortical tissue to various illumination conditions and then quantifying any changes in microglia morphology over the duration of light exposure or using immunohistochemistry after imaging. Generally, fluorescence imaging should be conducted using as little light exposure as possible.

\section{Data Analysis and Applications of Two-Photon Imaging}

Data analysis can be performed using public domain image processing software (e.g., ImageJ) or commercial software packages (Imaris from Bitplane). To study the interaction of microglia with other cellular elements in the brain, a variety of fluorescent markers and minimally invasive labeling approaches are available (Nimmerjahn et al. 2008). Tail vein injection of dextran-conjugated fluorescent dyes can be used to visualize blood vessels and blood-brain barrier leaks in the brain (Nimmerjahn et al. 2005; Bolmont et al. 2008; Koenigsknecht-Talboo et al. 2008). Co-staining of parenchymal cells other than microglia can be achieved by crossbreeding different transgenic mouse lines (Kim and Dustin 2006; Wake et al. 2009) (Figs. 2 and 3; see Movies 1 and 4 online at http:// cshprotocols.cshlp.org). In mouse models of Alzheimer's disease, amyloid can be stained by intraperitoneal injection of blood-brain barrier-permeable methoxy-X04 (Bolmont et al. 2008; Koenigsknecht-Talboo et al. 2008). In the future, expression of genetically encoded calcium indicators in microglia may allow study of their calcium signaling.

\section{RECIPE}

\section{ACSF for Two-Photon Imaging}

$125 \mathrm{~mm} \mathrm{NaCl}$
$5 \mathrm{~mm} \mathrm{KCl}$
$10 \mathrm{~mm}^{- \text {-glucose }}$
$10 \mathrm{~mm} \mathrm{HEPES}$
$2 \mathrm{mM} \mathrm{MgSO}_{4}$
$2 \mathrm{~mm} \mathrm{CaCl}_{2}$
Adjust pH to $\sim 7.3$ with $\mathrm{NaOH}$. Osmolarity should be $\sim 300$. 
The author thanks Richard Luong, Alessio Attardo, Jay Jiang, and Jill Helms for helpful discussions; Mark Schnitzer and Ben Barres for generous support; and Frank Kirchhoff for transgenic mice. This work was supported by postdoctoral fellowships to A.N. from the Alexander von Humboldt-Foundation and the International Human Frontier Science Program Organization.

\section{REFERENCES}

Bacskai BJ, Kajdasz ST, Christie RH, Carter C, Games D, Seubert P, Schenk D, Hyman BT. 2001. Imaging of amyloid-b deposits in brains of living mice permits direct observation of clearance of plaques with immunotherapy. Nat Med 7: 369-372.

Bolmont T, Haiss F, Eicke D, Radde R, Mathis CA, Klunk WE, Kohsaka S, Jucker M, Calhoun ME. 2008. Dynamics of the microglial/amyloid interaction indicate a role in plaque maintenance. J Neurosci 28: 4283-4292.

Christie RH, Bacskai BJ, Zipfel WR, Williams RM, Kajdasz ST, Webb WW, Hyman BT. 2001. Growth arrest of individual senile plaques in a model of Alzheimer's disease observed by in vivo multiphoton microscopy. $J$ Neurosci 21: 858-864.

Davalos D, Grutzendler J, Yang G, Kim JV, Zuo Y, Jung S, Littman DR, Dustin ML, Gan WB. 2005. ATP mediates rapid microglial response to local brain injury in vivo. Nat Neurosci 8: 752-758.

Dombeck DA, Khabbaz AN, Collman F, Adelman TL, Tank DW. 2007. Imaging large-scale neural activity with cellular resolution in awake, mobile mice. Neuron 56: 43-57.

Donnert G, Eggeling C, Hell SW. 2009. Triplet-relaxation microscopy with bunched pulsed excitation. Photochem Photobiol Sci 8: 481-485.

Engelbrecht CJ, Göbel W, Helmchen F. 2009. Enhanced fluorescence signal in nonlinear microscopy through supplementary fiber-optic light collection. Opt Express 17: 6421-6435.

Garcia-Alloza M, Bacskai BJ. 2004. Techniques for brain imaging in vivo. Neuromolecular Med 6: 65-78.

Greenberg DS, Kerr JN. 2009. Automated correction of fast motion artifacts for two-photon imaging of awake animals. J Neurosci Methods 176: $1-15$.

Grutzendler J, Kasthuri N, Gan WB. 2002. Long-term dendritic spine stability in the adult cortex. Nature 420: 812-816.

Haynes SE, Hollopeter G, Yang G, Kurpius D, Dailey ME, Gan WB, Julius D. 2006. The $\mathrm{P}_{2} \mathrm{Y}_{12}$ receptor regulates microglial activation by extracellular nucleotides. Nat Neurosci 9: 1512-1519.

Helm PJ, Ottersen OP, Nase G. 2009. Analysis of optical properties of the mouse cranium-Implications for in vivo multiphoton laser scanning microscopy. J Neurosci Methods 178: 316-322.

Helmchen F, Denk W. 2005. Deep tissue two-photon microscopy. Nat Methods 2: 932-940.

Holtmaat A, Bonhoeffer T, Chow DK, Chuckowree J, De Paola V, Hofer SB, Huebener M, Keck T, Knott G, Lee W-CA, et al. 2009. Long-term, highresolution imaging in the mouse neocortex through a chronic cranial window. Nat Protoc 4: 1128-1144.

Hopt A, Neher E. 2001. Highly nonlinear photodamage in two-photon fluorescence microscopy. Biophys J 80: 2029-2036.

Ji N, Magee JC, Betzig E. 2008. High-speed, low-photodamage nonlinear imaging using passive pulse splitters. Nat Methods 5: 197-202.

Kawamura S, Schuerer L, Goetz A, Kempski O, Schmucker B, Baethmann A. 1990. An improved closed cranial window technique for investigation of blood-brain barrier function and cerebral vasomotor control in the rat. Int J Microcirc Clin Exp 9: 369-383.

Kim JV, Dustin ML. 2006. Innate response to focal necrotic injury inside the blood-brain barrier. J Immunol 177: 5269-5277.

Koenigsknecht-Talboo J, Meyer-Luehmann M, Parsadanian M, GarciaAlloza M, Finn MB, Hyman BT, Bacskai BJ, Holtzman DM. 2008. Rapid microglial response around amyloid pathology after systemic anti-A $\beta$ antibody administration in PDAPP mice. $J$ Neurosci 28 14156-14164.

Koester HJ, Baur D, Uhl R, Hell SW. 1999. $\mathrm{Ca}^{2+}$ fluorescence imaging with pico- and femtosecond two-photon excitation: Signal and photodamage. Biophys J 77: 2226-2236.
Lalancette-Hebert M, Phaneuf D, Soucy G, Weng YC, Kriz J. 2009. Live imaging of Toll-like receptor 2 response in cerebral ischaemia reveals a role of olfactory bulb microglia as modulators of inflammation. Brain 132: $940-954$

Mandrekar S, Jiang QG, Lee CYD, Koenigsknecht-Talboo J, Holtzman DM, Landreth GE. 2009. Microglia mediate the clearance of soluble Ab through fluid phase macropinocytosis. J Neurosci 29: 4252-4262.

Navari RM, Wei EP, Kontos HA, Patterson JL. 1978. Comparison of the open skull and cranial window preparations in the study of the cerebral microcirculation. Microvasc Res 16: 304-315.

Nguyen QT, Tsai PS, Kleinfeld D. 2006. MPScope: A versatile software suite for multiphoton microscopy. J Neurosci Methods 156: 351-359.

Nimmerjahn A. 2012a. Surgical implantation of a head plate in mice in preparation for in vivo two-photon imaging of microglia. Cold Spring Harb Protoc doi: 10.1101/pdb.prot069278.

Nimmerjahn A. 2012b. Optical window preparation for two-photon imaging of microglia in mice. Cold Spring Harb Protoc doi: 10.1101/ pdb.prot069286.

Nimmerjahn A, Kirchhoff F, Helmchen F. 2005. Resting microglial cells are highly dynamic surveillants of brain parenchyma in vivo. Science 308: 1314-1318.

Nimmerjahn A, Theer P, Helmchen F. 2008. Two-photon laser scanning microscopy. In Ultrashort laser pulses in biology and medicine (ed Braun M, Gilch P, Zinth W), pp. 29-51. Springer-Verlag, Heidelberg.

Nimmerjahn A, Mukamel EA, Schnitzer MJ. 2009. Motor behavior activates Bergmann glial networks. Neuron 62: 400-412.

Oheim M, Beaurepaire E, Chaigneau E, Mertz J, Charpak S. 2001. Twophoton microscopy in brain tissue: Parameters influencing the imaging depth. J Neurosci Methods 111: 29-37.

Raivich G, Bohatschek M, Kloss CUA, Werner A, Jones LL, Kreutzberg GW. 1999. Neuroglial activation repertoire in the injured brain: Graded response, molecular mechanisms, and cues to physiological function. Brain Res Rev 30: 77-105.

Ransohoff RM, Perry VH. 2009. Microglial physiology: Unique stimuli, specialized responses. Annu Rev Immunol 27: 119-145.

Rueckel M, Mack-Bucher JA, Denk W. 2006. Adaptive wavefront correction in two-photon microscopy using coherence-gated wavefront sensing. Proc Natl Acad Sci 103: 17137-17142.

Spiess E, Bestvater F, Heckel-Pompey A, Toth K, Hacker M, Stobrawa G, Feurer T, Wotzlaw C, Berchner-Pfannschmidt U, Porwol T, et al. 2005. Two-photon excitation and emission spectra of the green fluorescent protein variants ECFP, EGFP, and EYFP. J Microsc 217: 200-204.

Tang S, Krasieva TB, Chen Z, Tempea G, Tromberg BJ. 2006. Effect of pulse duration on two-photon excited fluorescence and second harmonic generation in nonlinear optical microscopy. J Biomed Opt 11: 020501 .

Wake H, Moorhouse AJ, Jinno S, Kohsaka S, Nabekura J. 2009. Resting microglia directly monitor the functional state of synapses in vivo and determine the fate of ischemic terminals. J Neurosci 29: 3974-3980.

Walter ML, Domes ME, Diller RA, Sproedt J, Joosten UH. 1999. Photoablation of bone by excimer laser radiation. Lasers Surg Med 25: $153-158$.

Wiart M, Davoust N, Pialat J-B, Desestret V, Moucharrafie S, Cho T-H, Mutin M, Langlois J-B, Beuf O, Honnorat J, et al. 2006. MRI monitoring of neuroinflammation in mouse focal ischemia. Stroke 38: 131-137.

Xu HT, Pan F, Yang G, Gan WB. 2007. Choice of cranial window type for in vivo imaging affects dendritic spine turnover in the cortex. Nat Neurosci 10: $549-551$ 


\section{Two-Photon Imaging of Microglia in the Mouse Cortex In Vivo}

Axel Nimmerjahn

Cold Spring Harb Protoc; doi: 10.1101/pdb.prot069294

\begin{tabular}{|c|c|}
\hline $\begin{array}{r}\text { Email Alerting } \\
\text { Service }\end{array}$ & Receive free email alerts when new articles cite this article - click here. \\
\hline $\begin{array}{l}\text { Subject } \\
\text { Categories }\end{array}$ & $\begin{array}{l}\text { Browse articles on similar topics from Cold Spring Harbor Protocols. } \\
\text { Cell Imaging (525 articles) } \\
\text { Fluorescent Proteins ( } 259 \text { articles) } \\
\text { Imaging for Neuroscience ( } 342 \text { articles) } \\
\text { In Vivo Imaging ( } 334 \text { articles) } \\
\text { Mouse (437 articles) } \\
\text { Multi-Photon Microscopy (103 articles) } \\
\text { Transgenic Mice (139 articles) } \\
\text { Video Imaging / Time Lapse Imaging (171 articles) }\end{array}$ \\
\hline
\end{tabular}

